Discrete Comput Geom 27:155-161 (2002)

DOI: $10.1007 / \mathrm{s} 00454-001-0058-3$

Geometry

\title{
Circuit Admissible Triangulations of Oriented Matroids
}

\author{
J. Rambau \\ Konrad-Zuse-Zentrum für Informationstechnik Berlin, \\ Takustraße 7, 14195 Berlin, Germany \\ rambau@zib.de
}

\begin{abstract}
All triangulations of euclidean oriented matroids are of the same PL-homeomorphism type by a result of Anderson. That means all triangulations of euclidean acyclic oriented matroids are PL-homeomorphic to PL-balls and that all triangulations of totally cyclic oriented matroids are PL-homeomorphic to PL-spheres. For non-euclidean oriented matroids this question is wide open.

One key point in the proof of Anderson is the following fact: for every triangulation of a euclidean oriented matroid the adjacency graph of the set of all simplices "intersecting" a segment $\left[p_{-} p_{+}\right]$is a path. We call this graph the $\left[p_{-} p_{+}\right]$-adjacency graph of the triangulation.

While we cannot solve the problem of the topological type of triangulations of general oriented matroids we show in this note that for every circuit admissible triangulation of an arbitrary oriented matroid the $\left[p_{-} p_{+}\right]$-adjacency graph is a path.
\end{abstract}

Triangulations of oriented matroids appeared in the literature as natural combinatorial models for triangulations of point configurations [2]. However, since not all oriented matroids model point configurations the notion of a triangulation of an oriented matroid gives rise to additional questions that do not come up in the theory of triangulations of point configurations.

One of these questions is the following: is the abstract simplicial complex defined by a triangulation of an oriented matroid homeomorphic to a sphere in the totally cyclic case or a ball in the acyclic case? The answer to this question in the realizable case is of course affirmative because in the case of point configurations the triangulation is naturally embedded as a convex set in a euclidean space.

Why care about the general case? An application of triangulations of oriented matroids in their full generality is their appearance in the theory of combinatorial differential manifolds. "Good" topological properties in this context lead to the existence of differentiable structures on these objects, making the combinatorial model more suitable [1], [4]. 
However, also as an investigation of what weird things might happen in the theory of non-realizable oriented matroids this question has become a challenging open problem in its own right. (An in-depth study of triangulations of oriented matroids is presented in [5], background on oriented matroids can be found in [3].)

For a euclidean oriented matroid Anderson has proved that the topological types of all its triangulations are the same. Since for all oriented matroids there are triangulations known that are homeomorphic to a sphere (resp. to a ball)—the so-called lifting triangulations - the answer to the above question is affirmative for euclidean oriented matroids.

One important building block in the construction of Anderson is the fact that the adjacency graph of the set of simplices in a triangulation "intersecting" an arbitrary segment is always a path. (For exact definitions see below.)

In this note we show that this graph is also a path for general oriented matroids provided the triangulation respects an additional property: it does not contain a so-called intersection circuit, a circuit whose positive part is contained in one simplex and whose negative part is contained in another simplex of the triangulation.

We start by defining our main object of study. For simplicity, we call $r$-subsets of full rank $r$ simplices; $(r-1)$-subsets of rank $r-1$ are called facets of simplices (or facets, for short, whenever no confusion may arise). Recall that a facet of an oriented matroid is the complement of a positive cocircuit and may have more than $(r-1)$ elements. We therefore call facets of an oriented matroid OM-facets, for short.

Definition 1 (Circuit Admissible Triangulation). Let $\mathfrak{M}=(E, \mathcal{Z})$ be a rank $r$ oriented matroid on the ground set $E$ given by its set of circuits $\mathcal{Z}$. The set of OM-facets of $\mathfrak{M}$ be denoted by $\mathcal{L}(\mathfrak{M})$.

A non-empty set $T$ of simplices is called a circuit admissible triangulation of $\mathfrak{M}$ if it satisfies the following conditions:

(UP) For every $S \in T$ and every facet $R \subset S$ of $S$ either there is an OM-facet $F \in \mathcal{L}(\mathfrak{M})$ of $\mathfrak{M}$ with $R \subseteq F$ or there is another simplex $S^{\prime} \in T$ different from $S$ with $R \subset S^{\prime}$ (union property).

(IP) For all $S_{1}, S_{2} \in T$ there is no circuit $Z \in \mathcal{Z}$ with $Z^{+} \subseteq S_{1}$ and $Z^{-} \subseteq S_{2}$ (intersection property).

If two simplices or facets of simplices satisfy (IP) we call them circuit admissible. We follow the suggestion of Santos [5] to call a circuit violating (IP) an intersection circuit of the simplices involved.

Condition (UP) makes sure that there are enough simplices to "cover" the whole oriented matroid, (IP) takes care of unwanted intersections between simplices.

Recall that the definiton of a triangulation of an oriented matroid from Definition 2.1 of [5] coincides with Definition 1 concerning Condition (UP); Condition (IP) in Definition 1 is a strengthening of the original condition (called proper intersection), which reads as follows:

$\left(\mathrm{IP}^{\prime}\right)$ For every interior extension $p$ of $\mathfrak{M}$ with $p \in \operatorname{conv}_{\mathfrak{M} \cup p}\left(S_{1}\right) \cap \operatorname{conv}_{\mathfrak{M} \cup p}\left(S_{2}\right)$ for some $S_{1}, S_{2} \in T$ also $p \in \operatorname{conv}_{\mathfrak{M} \cup p}\left(S_{1} \cap S_{2}\right)$ must hold. 
In the euclidean case (IP) is equivalent to ( $\left.\mathrm{IP}^{\prime}\right)$. In general, Condition $\left(\mathrm{IP}^{\prime}\right)$ is - at least locally-weaker: the author has shown earlier (unpublished) an example in rank four based on the non-euclidean 12-point oriented matroid R(12) by Richter-Gebert where two simplices might intersect properly although they contain an intersection circuit.

Meanwhile, Santos has shown that this behavior already happens in the eight-point non-euclidean oriented matroid $\operatorname{EFM}(8)$ [5], the first case in which non-euclidean oriented matroids can appear. This shows a defect in the original definition of triangulations of oriented matroids by Billera and Munson [2]: proper intersection of simplices, although stated locally for two simplices, depends heavily on the rest of the oriented matroid via the existence of certain extensions. For example, the two simplices intersecting properly in $\mathrm{R}(12)$ but containing an intersection circuit would no longer intersect properly after suitable deletions, which seems somewhat unnatural. However, by Santos's work [5] we know that there are definitions equivalent to the original one that do not have this unwanted property.

The advantage of circuit admissability is the fact that an intersection of two simplices is not affected by elements that are not contained in the union of the two simplices involved. Note that every circuit admissible triangulation is a triangulation and that every triangulation additionally satisfying (IP) is a circuit admissible triangulation. Moreover, all lifting triangulations are easily seen to satisfy (IP); thus, there are circuit admissible triangulations available for every oriented matroid.

Here is a formal definition of our target:

Definition 2 (Segment Adjacency Part/Graph). Let $T$ be a triangulation of $\mathfrak{M}$, and let $p_{-}$and $p_{+}$be compatible interior extensions in general position.

The $\left[p_{-} p_{+}\right]$-adjacency part of $T$ is the set of all simplices $S$ of $T$, together with their pairwise common facets, that form a vector with the segment $\left[p_{-}, p_{+}\right]$(the restriction of $\mathfrak{M} \cup\left\{p_{-}, p_{+}\right\}$to $\left.\left\{p_{-}, p_{+}\right\}\right)$or with one of its endpoints, i.e., simplices or facets $S$ such that $\left(S, p_{-} p_{+}\right),\left(S, p_{-}\right)$, or $\left(S, p_{+}\right)$are vectors in $\mathfrak{M}$.

The $\left[p_{-}, p_{+}\right]$-adjacency graph $G_{\left[p_{-}, p+\right]}$ of $T$ is the adjacency graph of the $\left[p_{-} p_{+}\right]-$ adjacency part. In other words, it is the following graph:

- The vertex set of $G_{\left[p_{-}, p+\right]}$ is the set of all simplices $S$ in the $\left[p_{-} p_{+}\right]$-adjacency part.

- There is an edge between two vertices $S_{1}$ and $S_{2}$ if the corresponding simplices are adjacent, i.e., share a common facet.

We speak of the segment adjacency part (resp. segment adjacency graph) when we do not want to specify the segment explicitly.

Figure 1 depicts an easy example of a triangulation and a segment adjacency graph in the realizable case.

The following lemma was proved by Anderson for arbitrary triangulations of $e u$ clidean oriented matroids in a slightly different language.

Lemma 3 [1]. Let $T$ be a triangulation of a euclidean oriented matroid $\mathfrak{M}$ and let $p_{-}$ and $p_{+}$be interior extensions in general position. Then the $\left[p_{-}, p_{+}\right]$-adjacency graph of $T$ is a path. 


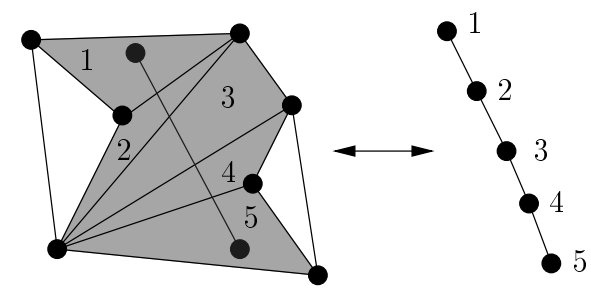

Fig. 1. The segment adjacency graph.

We should note the following at this point: because the restriction of $\mathfrak{M} \cup\left\{p_{-}, p_{+}\right\}$ to a segment plus a simplex is always realizable every node in $G_{\left[p_{-}, p+\right]}$ has degree one or two. Hence, the graph consists of one path and maybe some cycles. The point is to show that there are no cycles.

Lemma 4 (Main Lemma). Let $T$ be a circuit-admissible triangulation of $\mathfrak{M}$ and let $p_{-}$ and $p_{+}$be interior extensions in general position. Then the $\left[p_{-}, p_{+}\right]$-adjacency graph of $T$ is a path.

The idea for the proof is based on the following consideration: in the euclidean case we can extend the oriented matroid by the intersection points of facets with the segment $\left[p_{-} p_{+}\right]$. By looking at their cocircuit signature we can tell a total order on these intersection points, thus on the facets involved. The existence of cycles is incompatible with this observation.

In the non-euclidean case we might not be able to extend $\mathfrak{M}$ by all those intersection points. Thus, we do not have a total order of the facets intersecting a segment at hand. We can, however, replace the total order by a certain weaker relation. This relation will be good enough to prove the Main Lemma. The following definition is the key idea in this note.

Definition 5 (Segment Relation). Let $T$ be a circuit-admissible triangulation of $\mathfrak{M}$. For interior extensions $p_{-}$and $p_{+}$in general position, we define a relation on the facets in the $\left[p_{-}, p_{+}\right]$-adjacency part of $T$ as follows:

$F \prec_{\left[p_{-} p_{+}\right]} G \quad: \Longleftrightarrow \quad \exists$ circuit $X$ in $\mathfrak{M}: \quad p_{-} \in X^{+}, \quad X^{+} \subseteq p_{-} \cup G, \quad X^{-} \subseteq F$;

$F \succ_{\left[p_{-} p_{+}\right]} G \quad: \Longleftrightarrow \quad \exists$ circuit $X$ in $\mathfrak{M}: \quad p_{+} \in X^{+}, \quad X^{+} \subseteq p_{+} \cup G, \quad X^{-} \subseteq F$.

See Fig. 2 for an illustration in rank four.

This relation cannot provide a total order on the facets intersecting a segment because this would contradict the existence of cycling oriented matroid programs, a key property of non-euclidean oriented matroids. The following lemma shows, however, that the segment relation is good enough to tell consistently which one of two circuit admissible facets is "closer" to $p_{-}$and which one is "closer" to $p_{+}$. We use the notation $Z=$ $\left(Z^{+}, Z^{-}\right)$for a circuit and $\underline{Z}=Z^{+} \cup Z^{-}$for its support. 


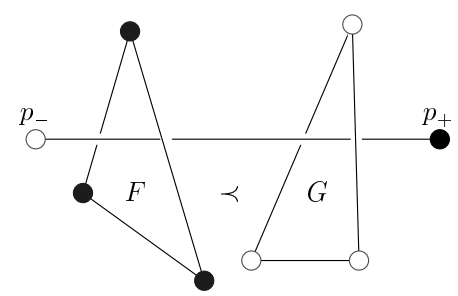

Fig. 2. The segment relation: $F \prec_{\left[p_{-} p_{+}\right]} G$ because there is a circuit containing $p_{-}$with a light positive and a dark negative part.

Lemma 6. Let $T$ be triangulation of $\mathfrak{M}$. Furthermore, let $F$ and $G$ be facets in the $\left[p_{-}, p_{+}\right]$-adjacency part of $T$ for interior extensions $p_{-}$and $p_{+}$in general position. Then the following hold:

(i) $F \prec_{\left[p_{-} p_{+}\right]} G$ if and only if $G \succ_{\left[p_{-} p_{+}\right]} F$.

(ii) If $T$ is circuit-admissible, then either $F \prec_{\left[p_{-} p_{+}\right]} G$ or $F \succ_{\left[p_{-} p_{+}\right]} G$, but not both.

Proof. All assertions follow easily from strong circuit elimination arguments.

To prove (i), let $F \prec_{\left[p_{-} p_{+}\right]} G$, i.e., there is a circuit $X$ with

$$
p_{-} \in X^{+} ; \quad X^{+} \subseteq p_{-} \cup G ; \quad X^{-} \subseteq F
$$

Using the circuit

$$
Y:=\left(G, p_{-} p_{+}\right)
$$

eliminate $p_{-} \in X^{+} \cap Y^{-}$and introduce $p_{+} \in \underline{Y} \backslash \underline{X}$. This yields a circuit $Z$ with

$$
\begin{aligned}
& p_{+} \in Z^{-} ; \\
& Z^{+} \subseteq\left(X^{+} \cup Y^{+}\right) \backslash p_{-} \subseteq G ; \\
& Z^{-} \subseteq\left(X^{-} \cup Y^{-}\right) \backslash p_{-} \subseteq p_{+} \cup F .
\end{aligned}
$$

Thus, $G \succ_{\left[p_{-} p_{+}\right]} F$. Since the statement is symmetric, the first claim is proved.

In order to prove (ii), we first show that at most one of the relations $F \prec_{\left[p_{-} p_{+}\right]} G$ and $F \succ_{\left[p_{-} p_{+}\right]} G$ holds. To this end, assume-for the sake of contradiction-that $F \prec_{\left[p_{-} p_{+}\right]}$ $G$ and $F \succ_{\left[p_{-} p_{+}\right]} G$. Using (i) we also have $G \prec_{\left[p_{-} p_{+}\right]} F$. By definition, there are circuits $X$ and $Y$ with

$$
\begin{array}{lll}
p_{-} \in X^{+} ; & X^{+} \subseteq p_{-} \cup G ; & X^{-} \subseteq F ; \\
p_{-} \in Y^{+} ; & Y^{+} \subseteq p_{-} \cup F ; & Y^{-} \subseteq G .
\end{array}
$$

Elimination of $p_{-} \in X^{+} \cap(-Y)^{-}$in $X$ yields a circuit $Z$ with

$$
\begin{aligned}
& Z^{+} \subseteq\left(X^{+} \cup(-Y)^{+}\right) \backslash p_{-} \subseteq G ; \\
& Z^{-} \subseteq\left(X^{-} \cup(-Y)^{-}\right) \backslash p_{-} \subseteq F .
\end{aligned}
$$

In other words, $F$ and $G$ are not circuit admissible. Consequently, $F \prec_{\left[p_{-} p_{+}\right]} G$ and $F \succ_{\left[p_{-} p_{+}\right]} G$ cannot hold at the same time. 
We finally show that at least one of the relations $F \prec_{\left[p_{-} p_{+}\right]} G$ or $F \succ_{\left[p_{-} p_{+}\right]} G$ holds. Since $F$ and $G$ are in the $\left[p_{-}, p_{+}\right]$-adjacency part, we have two circuits $X:=\left(F, p_{-} p_{+}\right)$ and $Y:=\left(G, p_{-} p_{+}\right)$. Eliminating $p_{-}$yields a circuit $Z$ with

$$
\begin{aligned}
& Z^{+} \subseteq\left(X^{+} \cup(-Y)^{+}\right) \backslash p_{-} \subseteq F \cup p_{+} ; \\
& Z^{-} \subseteq\left(X^{-} \cup(-Y)^{-}\right) \backslash p_{-} \subseteq G \cup p_{+} .
\end{aligned}
$$

If $p_{+} \notin \underline{Z}$, then $F$ and $G$ are not circuit admissible: contradiction. If $p_{+} \in Z^{+}$, then $Z$ is a circuit showing $G \succ_{\left[p_{-} p_{+}\right]} F$; if $p_{+} \in Z^{-}$, then $Z$ shows $F \succ_{\left[p_{-} p_{+}\right]} G$. This proves the claim using (i) one more time.

We are now in a position to prove our lemma.

Proof of Lemma 4. Since $T$ is a triangulation of $\mathfrak{M}$ and $p_{-}$and $p_{+}$are in general position it follows from the work of Santos [5] that there is a unique simplex $S_{+}$"containig" $p_{+}$, i.e., such that $\left(S_{+}, p_{+}\right)$is a vector, and a unique simplex $S_{-}$"containing" $p_{-}$, i.e., such that $\left(S_{-}, p_{-}\right)$is a vector in $\mathfrak{M}$.

From the fact that $\mathfrak{M}$ restricted to a segment plus one simplex is realizable it follows that $S_{-}$and $S_{+}$have degree one in $G_{\left[p_{-}, p_{+}\right]}$whereas all other simplices have degree two. Hence, there is a path from $S_{-}$to $S_{+}$contained in $G_{\left[p_{-}, p_{+}\right]}$. We have to show that all simplices in the $\left[p_{-}, p_{+}\right]$-adjacency part belong to that path.

Let $S_{0}=S_{-}, S_{1}, \ldots, S_{k}=S_{+}$be the successively adjacent simplices in the path from $S_{-}$to $S_{+}$. Assume, for the sake of contradiction, that there exists another simplex $S$ in $T$ with $S \neq S_{i}$ for all $i=1, \ldots, k$. Define $F_{j}:=S_{j-1} \cap S_{j}$ for $j=1, \ldots, k$. Furthermore, let $F$ be a facet of $S$ "pierced" by $\left[p_{-} p_{+}\right]$, i.e., so that $\left(F, p_{-} p_{+}\right)$is a circuit.

By Lemma 6, there are three cases:

- $F \prec_{\left[p_{-} p_{+}\right]} F_{1}$,

- $F \succ_{\left[p_{-} p_{+}\right]} F_{k}$, or

- $F_{i} \prec_{\left[p_{-} p_{+}\right]} F \prec_{\left[p_{-} p_{+}\right]} F_{i+1}$ for some $i \in\{1,2, \ldots, k-1\}$.

The case $F \prec_{\left[p_{-} p_{+}\right]} F_{1}$. Then there exists a circuit $X$ with

$$
p_{-} \in X^{+} ; \quad X^{+} \subseteq p_{-} \cup F_{1} ; \quad X^{-} \subseteq F .
$$

Eliminate $p_{-} \in X^{+} \cap Y^{-}$using the circuit

$$
Y:=\left(S_{0}, p_{-}\right)
$$

This yields a circuit $Z$ with

$$
\begin{aligned}
& Z^{+} \subseteq\left(X^{+} \cup Y^{+}\right) \backslash p_{-} \subseteq F_{1} \\
& Z^{-} \subseteq\left(X^{-} \cup Y^{-}\right) \backslash p_{-} \subseteq F
\end{aligned}
$$

Thus, $F_{1}$ and $F$ are not circuit admissible, and neither are $S_{0}$ and $S$ : contradiction.

The case $F \succ_{\left[p_{-} p_{+}\right]} F_{k}$. This case is analogous to the previous one. 
The case $F_{i} \prec_{\left[p_{-} p_{+}\right]} F \prec_{\left[p_{-} p_{+}\right]} F_{i+1}$ for some $i=0, \ldots, k-1$. Then there exist circuits $X$ and $Y$ with

$$
\begin{array}{lrr}
p_{-} \in X^{+}, & X^{+} \subseteq p_{-} \cup F, & X^{-} \subseteq F_{i} ; \\
p_{-} \in-Y^{-}, & -Y^{-} \subseteq p_{-} \cup F_{i+1}, & -Y^{+} \subseteq F ;
\end{array}
$$

Eliminate $p_{-} \in X^{+} \cap-Y^{-}$. This yields a circuit $Z$ with

$$
\begin{aligned}
& Z^{+} \subseteq\left(X^{+} \cup-Y^{+}\right) \backslash p_{-} \subseteq F \\
& Z^{-} \subseteq\left(X^{-} \cup-Y^{-}\right) \backslash p_{-} \subseteq F_{i} \cup F_{i+1}
\end{aligned}
$$

However, by construction $F_{i} \cup F_{i+1}=S_{i}$, and thus $S$ and $S_{i}$ are not circuit admissible: contradiction.

\section{References}

1. Laura Anderson, Topology of combinatorial differential manifolds, Topology 38 (1999), 197-221.

2. Louis J. Billera and Beth Spellman Munson, Triangulations of oriented matroids and convex polytopes, SIAM Journal of Algebraic Discrete Methods 5 (1984), 515-525.

3. Anders Björner, Michel Las Vergnas, Bernd Sturmfels, Neil White, and Günter M. Ziegler, Oriented Matroids, Encyclopedia of Mathematics, vol. 46, Cambridge University Press, Cambridge, 1993.

4. Robert D. MacPherson, Combinatorial Differential Manifolds, Topological Methods in Modern Mathematics: A Symposium in Honor of John Milnor's Sixtieth Birthday, Stony Brook NY, 1991 (Lisa R. Goldberg and Anthon V. Phillips, eds.), Publish or Perish, Houston, TX, 1993, pp. 203-221.

5. Francisco Santos, Triangulations of oriented matroids, Preprint, Universidad de Cantabria, 2000, Memoirs of the AMS, to appear.

Received December 8, 2000, and in revised form May 23, 2001. Online publication November 7, 2001. 